\title{
La Enfermedad Mental en los Medios de Comunicación: Un Estudio Empírico en Prensa Escrita, Radio y Televisión
}

\section{Mental Illness in the Media: An Empirical Study in Press, Radio and Television}

\author{
Manuel Muñoz \\ Universidad Complutense de Madrid, \\ España \\ Ana Isabel Guillén \\ Universidad Complutense de Madrid, España
}
Universidad Complutense de Madrid, España

Eloísa Pérez-Santos

\author{
María Crespo \\ Universidad Complutense de Madrid, \\ España \\ Sara Izquierdo \\ Hospital La Paz, Madrid, España
}

Resumen. La percepción social de la enfermedad mental está teñida de una serie de estereotipos negativos y prejuicios que conducen a la discriminación y aumentan el sufrimiento de las personas que padecen un trastorno mental. En repetidas ocasiones se ha señalado el papel de los medios de comunicación como agentes estigmatizadores de gran repercusión y difusión social. El presente estudio pretende cubrir la escasez de trabajos que analizan las posibles tendencias estigmatizantes en los principales medios de comunicación españoles. Los resultados muestran una utilización abusiva e incorrecta de terminología relacionada con los trastornos mentales en los contenidos informativos de prensa, radio y televisión, mientras que el seguimiento real del tema es escaso y se aleja de un tratamiento informativo óptimo. De hecho, una cuarta parte de las noticias analizadas incluían contenidos estigmatizadores, siendo los estereotipos con mayor frecuencia de aparición el de peligrosidad e impredecibilidad.

Todo ello hace necesaria la puesta en marcha de iniciativas de sensibilización social y erradicación del estigma, con el fin de favorecer la integración y el bienestar de las personas que sufren algún trastorno mental.

Palabras clave: estigma, trastorno mental, estereotipo, discriminación, medios de comunicación.

\begin{abstract}
The social perception of mental illness is characterised by a wide range of negative stereotypes and prejudices which lead to discrimination and increase the suffering of people with a mental disorder. The media have been repeatedly described as important stigmatizing agents with enormous social influence and repercussion. This study intends to compensate for the lack of investigations about possible stigmatizing trends in the main Spanish media. The results have shown an abusive and improper use of terminology related to mental disorders in the radio, television and press news reports, which contrasts with a lack of news stories which really dealt with the subject and offered high quality informa-
\end{abstract}

La correspondencia sobre este artículo deben enviarse al primer autor a la Facultad de Psicología, UCM. Campus de Somosaguas, s/n. 28223 Madrid (SPAIN). E-mail: mmunoz@psi.ucm.es
Agradecimientos: El presente estudio ha sido financiado por la Obra social de Caja Madrid y la Consejería de Familia y Asuntos Sociales de la Comunidad de Madrid. 
tion. In fact, a quarter of the analyzed news stories included stigmatizing contents, and a significant presence of stereotypes concerning danger and unpredictability.

This reality requires the setting up of social sensitization and stigma eradication campaigns, aimed at promoting the integration and well-being of those people suffering from a mental disorder.

Keywords: stigma, mental disorder, stereotype, discrimination, media.

\section{Introducción}

Una importante parte del sufrimiento de las personas con un trastorno mental tiene su origen en el proceso de estigmatización que, con frecuencia, acompaña a esta condición. Se trata de un conjunto de reacciones sociales negativas que limitan el bienestar, adaptación e integración social de quienes padecen una enfermedad mental (especialmente de carácter grave y crónico), afectando también a sus familiares y los profesionales de la salud mental (Muñoz, Pérez-Santos, Crespo y Guillén, 2009). Crocker, Major y Steele (1998) han señalado que los individuos estigmatizados poseen algún atributo o característica que conlleva una identidad social, la cual es devaluada en un contexto social particular. El estigma se manifiesta en tres niveles del comportamiento social (Ottati, Bodenhausen y Newman, 2004): estereotipos, prejuicios y discriminación.

Los estereotipos son creencias aprendidas que representan el acuerdo generalizado sobre lo que caracteriza a un determinado grupo. En el caso de la enfermedad mental, los estereotipos más habituales se refieren a la peligrosidad, violencia, impredecibilidad y debilidad de carácter, además de la responsabilidad sobre la enfermedad y la incompetencia para tareas básicas como el autocuidado (Crespo, Muñoz, Pérez-Santos y Guillén, 2008). Cuando, al aplicar estos estereotipos, una persona experimenta miedo, desconfianza u otras reacciones emocionales negativas, se están poniendo en marcha las actitudes y valores que constituyen los prejuicios. Todo ello puede conducir a una discriminación efectiva, esto es, a comportamientos de rechazo que coloquen a las personas con un trastorno mental en situación de desventaja social. En definitiva, los estereotipos, prejuicios y discriminaciones asociadas a la enfermedad mental pueden privar a quienes la padecen de oportunidades esenciales para el logro de sus objetivos vitales, especialmente aquellos que tienen que ver con su independencia económica y personal (Corrigan, 2005; Thornicroft, 2006).

A partir del amplio conjunto de investigaciones dirigidas a evaluar el estigma entre quienes padecen enfermedad mental, se ha observado una tendencia a encontrar elevados porcentajes de participantes que creen que serán devaluados por el hecho de padecer un trastorno mental y anticipan vivencias estigmatizadoras de rechazo social (e.g., Link et al., 2001; Link et al., 1997; Markowitz, 1998; Ritsher y Phelan, 2004).

Este estigma percibido o anticipado resulta especialmente nocivo a nivel emocional y conductual. En concreto, se ha observado que guarda una correlación negativa con la calidad de vida (Markowitz, 1998; Rosenfield, 1997) y la autoestima (Link et al., 2001; Markowitz, 1998), mientras que se relaciona positivamente con síntomas de depresión y ansiedad (Ertugrul y Ulug, 2004; Link et al., 1997; Markowitz, 1998).

Los estudios también han encontrado altos niveles de estigma experimentado (Depla et al., 2005; Dickerson et al., 2002; Walh, 1999) y estigma internalizado o auto-estigma (Ritsher y Phelan, 2004), que constituyen un obstáculo real para la asistencia e integración social de las personas con trastornos mentales graves.

Con el fin de comprender el mantenimiento de la estigmatización de las personas con trastornos mentales en nuestra sociedad, es ineludible atender al papel de los medios de comunicación de masas, probablemente los mayores agentes cristalizadores y difusores de las distintas tendencias sociales, cuya capacidad de influencia se observa en su creciente peso en la formación de las percepciones y actitudes sociales (Cuenca, 2003).

De hecho, se ha subrayado la responsabilidad de los medios de comunicación en la perpetuación de estereotipos, prejuicios y conductas discriminatorias, como consecuencia de la representación nega- 
tiva e imprecisa que hacen de los trastornos mentales (Cutcliffe y Hannigan, 2001). No obstante, también hay autores que defienden que los medios son tan sólo un espejo de la realidad social y transmiten la imagen que está en la calle y los ciudadanos quieren leer, ver u oír (Sieff, 2003).

El papel de los medios de comunicación en este proceso de estigmatización, es examinado en un considerable número de trabajos, fundamentalmente fuera de nuestro país. A pesar de la falta de sistematización de los procedimientos y la consiguiente dificultad de comparación, es posible extraer algunas conclusiones generales:

La mayoría de las investigaciones se han centrado en estudiar la representación que hacen los medios de los trastornos mentales y la posible aparición de tendencias estigmatizadoras, principalmente en prensa escrita, seguida del cine, televisión y radio. En general, se ha encontrado que en la prensa escrita hay un escaso número de informaciones con referencia a la enfermedad mental, contrariamente a lo que sucede en los contenidos de ficción y entretenimiento de la televisión o el cine.

La representación de la enfermedad mental en la prensa es predominantemente negativa (e.g, Coverdale et al., 2002; Nairn, Coverdale y Claasen, 2001; Walh et al., 2002), apareciendo ligada con frecuencia a sucesos violentos y delictivos (e.g., Angermeyer y Schulze, 2001; Coverdale et al, 2002; Gamo, 1992; Huang y Pruebe, 2003; Instituto Andaluz de Salud Mental, 1987). Generalmente se destacan los aspectos negativos, con una escasa presencia de informaciones sobre personas con trastorno mental que llevan una vida normalizada. Es esta una visión en consonancia con los estereotipos de peligrosidad, impredecibilidad, falta de control o vulnerabilidad.

A pesar de la posible evolución positiva en el tipo de terminología empleada (Arceo, 2005; Coverdale et al., 2002) sigue siendo habitual encontrar referencias a trastornos mentales en términos coloquiales con connotaciones peyorativas (e.g., Lawson y Fouts, 2004; Signorelli, 1989; Walh et al., 2003). Además, la enfermedad mental suele aparecer citada de forma negativa y sin información adicional sobre síntomas, causas, procesos de rehabilitación y tratamientos (e.g., Angermeyer y Schulze, 2001; Gamo,
1992; Nairn et al., 2001). En este sentido, los hallazgos de Francis et al. (2004) resultan más bien una excepción, al encontrar que los medios de comunicación australianos ofrecen información abundante y de buena calidad sobre los trastornos mentales.

En nuestro país encontramos una gran ausencia de investigación en esta área, pudiendo referirnos únicamente a tres trabajos, conducidos por el Instituto Andaluz de Salud Mental de Andalucía (1987), Gamo (1992) y Arceo (2005). Sus conclusiones coinciden en señalar marcadas carencias y una manifiesta polarización hacia lo negativo en el tratamiento de la patología mental en la prensa escrita española, estando lejos de ofrecerse un tratamiento informativo de calidad.

Otras investigaciones se han dirigido a estudiar específicamente la relación entre el negativo retrato que hacen los medios de las personas con un trastorno mental y los estereotipos, prejuicios y conductas discriminatorias de la población (Angermeyer y Schulze, 2001; Angermeyer et al., 2005; Minnebo y Van Acker, 2004). De sus resultados parece deducirse que el tratamiento que los medios de comunicación otorgan a los trastornos mentales tiene una repercusión significativa en las percepciones y actitudes del público, conceptualizadas en forma de opiniones desfavorables y deseo de distancia social. Sin embargo, hemos de tener en cuenta que se trata de un reducido número de trabajos de carácter cuasiexperimental (no permiten establecer relaciones de carácter causal, sino correlacional) y con carencias metodológicas como la falta de medidas a largo plazo.

A medida que aumenta nuestro conocimiento, se hace evidente la necesidad de desarrollar iniciativas institucionales dirigidas a la promoción de la salud mental, la prevención de los trastornos mentales y la disminución del estigma (Asociación Española de Neuropsiquiatría, 2007; OMS, 2001). La información disponible es favorable a la aplicación de estrategias complejas basadas en difundir información apropiada, facilitar la interacción social de las personas con trastorno mental, favorecer acciones de protesta, eliminar factores de refuerzo del estigma y apoyar a las personas afectadas. (López et al., 2008). En consonancia con este modelo, se han puesto en marcha algunas iniciativas que se sirven de los 
medios de comunicación, consiguiendo aparentemente ciertos cambios en dirección a la reducción del estigma social (Vaughan y Hansen, 2004).

Un paso previo para el avance en la integración de las personas con enfermedad mental grave y crónica es profundizar en la comprensión del estigma social. El presente trabajo trata de examinar el tratamiento que se otorga a la enfermedad mental en los espacios informativos de los medios de comunicación de nuestro país y la posible presencia de tendencias estigmatizadoras. De este modo, se pretende compensar en algún grado la escasez de este tipo de estudios dentro de nuestras fronteras, así como el limitado número de trabajos que han contemplado diversos medios de comunicación al mismo tiempo (Francis et al., 2004; Francis et al., 2005).

\section{Método}

\section{Procedimiento de muestreo}

\section{Selección de medios}

El presente estudio trata de analizar las informaciones que llegan mayoritariamente a los ciudadanos, por lo que la selección se ha realizado en base a los datos de la Encuesta General de Medios correspondiente al periodo comprendido entre Abril de 2004 y Marzo de 2005 (Asociación para la investigación de Medios de Comunicación, 2005).

El análisis se circunscribe a los periódicos, la radio y la televisión, por ser los tres medios de comunicación más utilizados por la población para informarse.

En lo concerniente a la prensa escrita, se han incluido los tres periódicos nacionales de información general con mayor número de lectores diarios: El País, El Mundo y el ABC, analizando las ediciones correspondientes a Madrid por ser éste nuestro principal foco de interés.

Las emisoras de radio seleccionadas por encabezar el ranking de audiencia han sido la Cadena SER, la COPE y Onda Cero. Radio Nacional de España también fue incluida a pesar de no figurar en la Encuesta General de Medios por haberse retirado en 2003 de la asociación que lleva a cabo el estudio. No obstante, las cifras de la última encuesta de la que formó parte la situaban en tercer lugar según su número de oyentes diarios.

El equipo de investigación se puso en contacto con cada emisora para seleccionar el informativo y la franja horaria con mayor audiencia. Finalmente se incluyeron en el estudio:

- Hoy por Hoy (La SER, L-V, 07:00-08:30 h.).

- Matinal SER (La SER, Fines de semana, 08:0009:00).

- El Foro (La SER, L-V, 19:00-20:00. Elegida por ser el informativo específico de Madrid con mayor audiencia en la Comunidad).

- La mañana (La COPE, L-D, 07:00-08:30)

- La Brújula (Onda Cero, L-V 20-20:30 + 2222:30).

- España a las 8 (RNE1, L-V 08:00- 09:00/ Fines de Semana 08:00-08:15).

En el caso de la televisión se incluyeron los informativos de la noche (por ser los más seguidos) de las cadenas de mayor audiencia: Tele 5, Antena 3 y TVE 1, a las que se sumó también Telemadrid. Además, se incluyeron los programas Sucedió en Madrid (Telemadrid) y Gente (TVE1) por versar sobre noticias de sucesos, contenido frecuentemente ligado a estereotipos sobre la enfermedad mental.

\section{Periodo de muestreo}

El estudio se ha realizado sobre las informaciones trasmitidas durante 14 días seguidos, del Lunes 11 al Domingo 24 de Abril de 2005. Se puso atención al hecho de que fuese un periodo informativo "normal" ( $\sin$ incluir vacaciones o días festivos) en el que no estuviese previsto ningún acontecimiento susceptible de monopolizar los espacios informativos.

Este periodo de dos semanas se ha considerado suficientemente representativo para extraer conclusiones fiables sobre la realidad informativa de nuestro entorno.

\section{Unidad de análisis}

La unidad de análisis utilizada en este estudio es la "unidad informativa" o cualquier texto oral o 
escrito precedido por un titular, lo cual ha permitido incorporar diversas tipologías informativas como noticias, entrevistas, artículos de opinión, etc.

El principal parámetro de medida ha sido la frecuencia de unidades informativas, así como el tiempo de emisión en el caso de la radio y la televisión.

\section{Contenidos incluidos en el muestreo}

En lo concerniente a la prensa escrita, se analizaron prácticamente todos los contenidos de los periódicos seleccionados (exceptuando la publicidad, cartelera, programación de TV, cartas de los lectores, cotización de bolsa, esquelas, información metereológica, pasatiempos y suplementos especiales). En total se examinaron 7532 unidades informativas: 2529 del País, 2473 del Mundo y 2530 del ABC.

Respecto a los contenidos radiofónicos, se analizó un tiempo de emisión estimado de 3079'34 minutos, una vez descontada la publicidad y la información metereológica. De ellos, 537 correspondían a Hoy por Hoy (SER), 225'48 al Matinal SER, 341'37 al Foro (SER), 860'73 se emitieron en La mañana (COPE), 479'16 en La brújula (Onda Cero) y 635'60 en España a las 8 (RNE 1).

La estimación del tiempo se realizó cronometrando determinados días de emisión seleccionados aleatoriamente y extrapolando el resultado a la totalidad de los días de emisión. La medición de los programas diarios la Mañana y España a las 8 fue tomada dos días de entre semana y uno del fin de semana. En el caso de La Brújula, El foro y Hoy por Hoy, programas sin emisión durante los fines de semana, se cronometraron dos días. Por último, el programa de fin de semana de Matinal SER fue cronometrado en una de sus emisiones y el resultado se extrapoló a las cuatro totales.

Los espacios televisivos seleccionados se analizaron en su práctica totalidad, con la salvedad de los cortes publicitarios y la información metereológica. La estimación total del tiempo de emisión estudiado es de 2284'89 minutos. Esta estimación se realizó con el mismo procedimiento que el descrito para la radio.

En el caso de los informativos de Tele 5 (duración total estimada de 504'18 min.), Antena 3
(598'09 min.), TVE 1 (638'67 min.) y Telemadrid (430’68 min.) se midió su duración durante dos días laborables y dos de fin de semana, mientras que para los programas Gente (duración estimada de 44’01 min.) y Sucedió en Madrid (69’26 min.) se cronometró una emisión y el resultado se extrapoló a la otra.

\section{Análisis de datos}

Con el objetivo de profundizar en el análisis de la información recogida, se ha considerado preciso efectuar tres tipos de análisis descriptivos: clasificación de las unidades informativas, análisis de frecuencias y análisis de contenidos.

\section{Clasificación de las unidades informativas}

El proceso se inició con la selección de todas las unidades informativas de prensa escrita que contuviesen palabras incluidas dentro de una lista exhaustiva de 368 términos psicológicos y/o psiquiátricos o expresiones del lenguaje común habitualmente utilizadas para referirse a la salud mental de las personas.

Tras el escrutinio de estas unidades informativas se pudo descartar aquellas que no se ajustaban plenamente a los objetivos propuestos y establecer los criterios de selección definitivos que permitiesen el análisis de los contenidos de radio y televisión.

Las informaciones finalmente seleccionadas fueron clasificadas en cinco categorías. Teniendo en cuenta su orden ascendente, cuando una unidad informativa podía ser asignada a más de una categoría se optaba por encuadrarla en la de mayor rango:

- Categoría 0: Unidades informativas sin relación con la salud mental. Inicialmente seleccionadas pero descartadas en un segundo momento de análisis.

- Categoría A: Unidades informativas que incluyen términos o expresiones psicológicas aplicadas a situaciones u objetos.

- Categoría B: Unidades informativas que incluyen términos o expresiones psicológicas aplica- 
dos a personas o grupos, y unidades informativas sobre salud mental general.

- Categoría C: Unidades informativas que incluyen términos o expresiones relacionados con enfermedad mental crónica (esquizofrenia y otros cuadros psicóticos o sus síntomas) aplicadas a situaciones u objetos.

- Categoría D: Unidades informativas que incluyen términos o expresiones relacionados con enfermedad mental crónica (esquizofrenia y otros cuadros psicóticos o sus síntomas) aplicadas a personas o grupos y unidades informativas sobre enfermedad mental crónica.

\section{Análisis de frecuencias}

El estudio incluye un análisis descriptivo de frecuencias global, consistente en examinar el número de unidades informativas asignadas a cada categoría procedentes de cada periódico, programa de radio y programa de televisión. Además, se realizó un análisis de frecuencias en función de cada medio de comunicación.

En lo concerniente a la prensa, se analiza el número de unidades informativas asignadas a cada categoría y el porcentaje que representan del total y de forma separada por categorías y periódicos.

Los análisis de frecuencias en radio y televisión miden el tiempo dedicado a las unidades informativas asignadas a cada categoría y el porcentaje que suponen sobre el total de minutos de cada programa y sobre el total de tiempo de radio o televisión analizado.

\section{Análisis de contenido}

El siguiente paso de análisis de la información recogida implica examinar el contenido específico de las unidades informativas que incluyen términos o expresiones relacionadas con enfermedad mental crónica (categorías C y D) y que, por ello, suponen el principal foco de interés del presente estudio.

A este respecto, se siguió el siguiente esquema de análisis: a) Análisis de los términos empleados en relación a la enfermedad mental crónica.

b) Análisis de las secciones en que se ubican estas unidades informativas (Este análisis no es posible en el caso de la radio y la televisión, donde los contenidos no están claramente adscritos a secciones concretas)

c) Análisis temático de las unidades informativas.

d) Análisis de cada unidad informativa por parte de nueve jueces independientes, todos ellos licenciados en Psicología y especializados en Salud Mental. Los jueces debían leer la definición de estigma facilitada y de los estereotipos más frecuentemente asociados a la enfermedad mental, para posteriormente valorar seis aspectos de cada unidad informativa $\mathrm{C}$ y $\mathrm{D}$ en un formato de respuesta $\mathrm{Si} / \mathrm{No}$ :

- La presencia de información errónea sobre la enfermedad mental (Calidad de la información).

- Presencia del estereotipo de peligrosidad.

- Presencia del estereotipo de responsabilidad.

- Presencia del estereotipo de impredecibilidad.

- Presencia del estereotipo de incompetencia.

- Si la unidad informativa resulta estigmatizante.

\section{Resultados}

\section{Resultados del análisis de frecuencias}

\section{Frecuencias globales}

El recuento de unidades informativas asignadas a cada categoría en función del medio de comunicación concreto del que proceden se muestra en la tabla 1 .

La suma total de las unidades informativas en los periódicos es de 625 unidades, 409 si excluimos aquellas pertenecientes a la categoría 0 , que se descartaron por incluir términos psicológicos sin relación con la salud mental. La cifra se sitúa en 50 unidades informativas para el caso de la radio y 57 para la televisión. Observamos como el mayor número de unidades se engloba dentro de la categoría B, esto es, informaciones en las que aparecen términos psicológicos aplicados a personas y unidades informativas sobre salud mental general. 
Tabla 1. Número de unidades informativas de cada categoría en función del medio de comunicación

\begin{tabular}{|c|c|c|c|c|c|c|c|c|}
\hline & & & \multicolumn{5}{|c|}{ Categoría de la unidad informativa } & \multirow[b]{2}{*}{$\begin{array}{c}\text { Tota } \\
\mathbf{N}^{\mathbf{0}}\end{array}$} \\
\hline & & & $\begin{array}{c}\mathbf{0} \\
\mathbf{N}^{\mathbf{0}}\end{array}$ & $\begin{array}{c}\mathbf{A} \\
\mathbf{N}^{\mathbf{o}}\end{array}$ & $\begin{array}{c}\mathbf{B} \\
\mathbf{N}^{\mathbf{o}}\end{array}$ & $\begin{array}{c}\mathbf{C} \\
\mathbf{N}^{\mathbf{0}}\end{array}$ & $\begin{array}{c}\mathbf{D} \\
\mathbf{N}^{\mathbf{o}}\end{array}$ & \\
\hline PRENSA & El País & & 99 & 61 & 36 & 43 & 33 & 272 \\
\hline \multirow[t]{2}{*}{ ESCRITA } & El Mundo & & 30 & 27 & 31 & 48 & 36 & 172 \\
\hline & $A B C$ & & 87 & 24 & 25 & 28 & 17 & 181 \\
\hline \multirow[t]{5}{*}{ RADIO } & SER & Hoy por hoy. Matinal & - & 0 & 5 & 2 & 3 & 10 \\
\hline & & El Foro & - & 3 & 4 & 3 & 0 & 10 \\
\hline & COPE & La Mañana & - & 0 & 9 & 5 & 6 & 20 \\
\hline & Onda Cero & La Brújula & - & 0 & 2 & 1 & 2 & 5 \\
\hline & RNE 1 & España a las 8 & - & 1 & 2 & 0 & 2 & 5 \\
\hline \multirow[t]{7}{*}{ TV } & Tele 5 & Noticias 20:30 & - & 2 & 1 & 1 & 44 & 8 \\
\hline & Antena 3 & Noticias $21 \mathrm{~h}$. & - & 3 & 4 & 2 & 7 & 16 \\
\hline & TVE 1 & Telediario $21 \mathrm{~h}$ & - & 0 & 7 & 0 & 5 & 12 \\
\hline & & Gente & - & 0 & 2 & 0 & 0 & 2 \\
\hline & Telemadrid & Noticias 20:30 & - & 4 & 8 & 1 & 2 & 15 \\
\hline & & Sucedió en Madrid & - & 0 & 1 & 1 & 2 & 4 \\
\hline & & Total & 216 & 125 & 137 & 135 & 119 & 732 \\
\hline
\end{tabular}

Nota: 0= Unidades informativas que incluyen términos sin relación con la salud mental.; A: Incluyen términos psicológicos aplicados a situaciones u objetos.; B= Incluyen términos psicológicos aplicados a personas o versan sobre salud mental general; $\mathrm{C}=$ Incluyen términos relacionados con enfermedad mental crónica aplicados a situaciones u objetos; $\mathrm{D}=$ Incluyen términos relacionados con enfermedad mental crónica aplicados a personas o versan sobre enfermedad mental crónica.

\section{Frecuencias en prensa escrita}

Del total de 7532 unidades informativas analizadas en los periódicos, 409 pertenecen a las categorías $\mathrm{A}, \mathrm{B}, \mathrm{C}$ o D, lo que implica que el $5.43 \%$ de las noticias examinadas incluyen algún término psicológico o palabras del lenguaje común habitualmente utilizadas para referirse a la salud mental de las personas. El porcentaje de unidades informativas que hacen referencia a enfermedad mental grave y crónica se sitúa en el $2.72 \%$ (205 unidades informativas en las categorías C y D).

Concretamente, el mayor número de unidades se engloba dentro de la categoría C (119 unidades) seguido por la categoría A (112 unidades).

\section{Frecuencias en radio}

La tabla 2 recoge los resultados del análisis de frecuencias de la información emitida por radio. De las 50 noticias que incluyeron referencias a la salud mental, conformando únicamente un $2,2 \%$ de tiempo total de emisión, 24 contenían de forma específica términos relacionados con enfermedad mental grave y crónica. En concreto, la mayor parte del tiempo lo ocuparon unidades informativas pertenecientes a la categoría D.

\section{Frecuencias en televisión}

Tal y como refleja la tabla 3, el tiempo que los informativos televisivos dedican a contenidos incluidos en las categorías A, B, C y D representa el $4,24 \%$ del tiempo total analizado, pero el porcentaje desciende a un $2,05 \%$ si atendemos únicamente a las unidades informativas que hacen referencia específica a trastornos mentales de naturaleza grave y crónica..

Las cifras son más elevadas en lo concerniente a los programas de sucesos "Gente" y "Sucedió en Madrid", espacios televisivos que dedican un $22 \%$ del tiempo de emisión analizado a emitir unidades informativas que incluyen términos relacionados con la psicología o expresiones del lenguaje común para referirse a la salud mental. Durante 13,08 minutos o un $11,55 \%$ del tiempo total, se abordan temas relacionados con la enfermedad mental crónica. 
Tabla 2. Minutos de unidades informativas de cada categoría y porcentajes sobre el tiempo total de cada programa y el tiempo total analizado en radio (entre paréntesis)

\begin{tabular}{|c|c|c|c|c|c|c|}
\hline \multirow[t]{2}{*}{ CADENA } & \multirow[t]{2}{*}{ PROGRAMA } & \multicolumn{4}{|c|}{ Categoría de la Unidad Informativa } & \multirow{2}{*}{$\begin{array}{l}\text { TOTAL } \\
\min .(\%)\end{array}$} \\
\hline & & $\underset{\min .(\%)}{\mathbf{A}}$ & $\begin{array}{c}\mathbf{B} \\
\min .(\%)\end{array}$ & $\begin{array}{c}\mathbf{C} \\
\min .(\%)\end{array}$ & $\begin{array}{c}\mathbf{D} \\
\min .(\%)\end{array}$ & \\
\hline \multirow[t]{4}{*}{ SER } & Hoy por Hoy (diario) & .00 & 2.80 & 1.27 & 7.19 & 11.26 \\
\hline & $\begin{array}{l}\text { Matinal (fin de semana) } \\
-762,48 \text { min. }\end{array}$ & $(.00)$ & $(.37)$ & $(.17)$ & $(.94)$ & $(1.48)$ \\
\hline & El Foro & 3.35 & 15.30 & 4.82 & 0.00 & 23.47 \\
\hline & - 341, $37 \mathrm{~min}$. & $(0.98)$ & $(4.48)$ & $(1.41)$ & $(.00)$ & $(6.87)$ \\
\hline \multirow[t]{2}{*}{ COPE } & La mañana & .00 & 2.95 & 8.32 & 12.93 & 24.20 \\
\hline & $-860,73 \mathrm{~min}$ & $(.00)$ & $(0.34)$ & $(0.97)$ & $(1.50)$ & $(2.81)$ \\
\hline \multirow[t]{2}{*}{ Onda Cero } & La brújula & .00 & 0.73 & 0.47 & 1.93 & 3.13 \\
\hline & $479,16 \mathrm{~min}$. & $(.00)$ & $(0.15)$ & $(0.10)$ & $(0.40)$ & $(0.65)$ \\
\hline \multirow{3}{*}{ RNE 1} & España a las 8 & 0.98 & 1.97 & .00 & 2.81 & 5.76 \\
\hline & - 635,60 min. & $(0.15)$ & $(0.31)$ & $(.00)$ & $(0.44)$ & $(0.91)$ \\
\hline & $\begin{array}{l}\text { TOTAL } \\
-3079,34 \mathrm{~min} .\end{array}$ & $\begin{array}{l}4.33 \\
(.14)\end{array}$ & $\begin{array}{r}23.75 \\
(.77)\end{array}$ & $\begin{array}{r}14.88 \\
(.48)\end{array}$ & $\begin{array}{r}24.86 \\
(.81)\end{array}$ & $\begin{array}{l}67.82 \\
(2.20)\end{array}$ \\
\hline
\end{tabular}

Nota: $\mathrm{A}=$ Unidades informativas que incluyen términos psicológicos aplicados a situaciones u objetos.; $\mathrm{B}=$ Incluyen términos psicológicos aplicados a personas o versan sobre salud mental gene$\mathrm{ral}$; $\mathrm{C}=$ Incluyen términos relacionados con enfermedad mental crónica aplicados a situaciones u objetos; $\mathrm{D}=$ Incluyen términos relacionados con enfermedad mental crónica aplicados a personas o versan sobre enfermedad mental crónica.

Tabla 3. Minutos de unidades informativas de cada categoría y porcentajes sobre el tiempo total de cada programa y el tiempo total analizado en televisión (entre paréntesis)

\begin{tabular}{|c|c|c|c|c|c|c|}
\hline \multirow[t]{2}{*}{ CADENA } & \multirow[t]{2}{*}{ PROGRAMA } & \multicolumn{4}{|c|}{ Categoría de la Unidad Informativa } & \multirow{2}{*}{$\begin{array}{l}\text { TOTAL } \\
\min .(\%)\end{array}$} \\
\hline & & $\underset{\min .(\%)}{\mathbf{A}}$ & $\begin{array}{c}\text { B } \\
\min .(\%)\end{array}$ & $\begin{array}{c}\mathbf{C} \\
\min .(\%)\end{array}$ & $\begin{array}{c}\text { D } \\
\min .(\%)\end{array}$ & \\
\hline SER & Hoy por Hoy (diario) & .00 & 2.80 & 1.27 & 7.19 & 11.26 \\
\hline Tele 5 & $\begin{array}{l}\text { Noticias } 20: 30 \mathrm{~h} . \\
\text { - 504,18 min. }\end{array}$ & $\begin{array}{c}1.82 \\
(0.36)\end{array}$ & $\begin{array}{c}2.73 \\
(0.54)\end{array}$ & $\begin{array}{c}1.43 \\
(0.28)\end{array}$ & $\begin{array}{c}7.32 \\
(1.45)\end{array}$ & $\begin{array}{l}13.30 \\
(2.64)\end{array}$ \\
\hline Antena 3 & $\begin{array}{l}\text { Noticias } 21 \mathrm{~h} \text {. } \\
\text { - 598,09 min. }\end{array}$ & $\begin{array}{l}3.75 \\
(0.63)\end{array}$ & $\begin{array}{l}11.00 \\
(1.84)\end{array}$ & $\begin{array}{l}3.77 \\
(0.63)\end{array}$ & $\begin{array}{l}16.52 \\
(2.76)\end{array}$ & $\begin{array}{l}35.04 \\
(5.86)\end{array}$ \\
\hline TVE 1 & $\begin{array}{l}\text { Telediario } 21 \mathrm{~h} \text {. } \\
-638,67\end{array}$ & $\begin{array}{l}.00 \\
(.00)\end{array}$ & $\begin{array}{l}11.01 \\
(1.72)\end{array}$ & $\begin{array}{l}.00 \\
(.00)\end{array}$ & $\begin{array}{l}10.60 \\
(1.66)\end{array}$ & $\begin{array}{l}21.61 \\
(3.38)\end{array}$ \\
\hline Telemadrid & $\begin{array}{l}\text { Telenoticias 20:30 h. } \\
\text { - 430,68 min. }\end{array}$ & $\begin{array}{l}.00 \\
(.00)\end{array}$ & $\begin{array}{l}16.80 \\
(3.90)\end{array}$ & $\begin{array}{l}.70 \\
(.16)\end{array}$ & $\begin{array}{c}4.68 \\
(1.09)\end{array}$ & $\begin{array}{l}22.18 \\
(5.15)\end{array}$ \\
\hline & $\begin{array}{l}\text { TOTAL } \\
2171,62 \text { min. }\end{array}$ & $\begin{array}{c}5.57 \\
(0.26)\end{array}$ & $\begin{array}{c}41.54 \\
(1.91)\end{array}$ & $\begin{array}{c}5.90 \\
(0.27)\end{array}$ & $\begin{array}{l}39.12 \\
(1.80)\end{array}$ & $\begin{array}{l}92.13 \\
(4.24)\end{array}$ \\
\hline
\end{tabular}

Nota: A= Unidades informativas que incluyen términos psicológicos aplicados a situaciones u objetos.; B= Incluyen términos psicológicos aplicados a personas o versan sobre salud mental gene$\mathrm{ral} ; \mathrm{C}=$ Incluyen términos relacionados con enfermedad mental crónica aplicados a situaciones u objetos; $\mathrm{D}=$ Incluyen términos relacionados con enfermedad mental crónica aplicados a personas o versan sobre enfermedad mental crónica.

\section{Resultados del análisis de contenidos}

\section{Terminología}

Tras la realización de un exhaustivo listado y el recuento de frecuencias de los 116 términos y expresiones encontradas en las unidades informativas de las categorías $\mathrm{C}$ y $\mathrm{D}$, se observa que el término con mayor frecuencia de aparición es el de "locos" aplicado a objetos, situaciones o personas (en 107 oca- siones), seguido por los términos "locura" (48 apariciones) y "delirio" (28 ocasiones).

Con el fin de profundizar en este análisis, se realizó una clasificación de los términos del listado inicial en 16 grupos. La tabla 4 recoge la frecuencia y proporción de unidades informativas con términos pertenecientes a estos 16 grupos en función de la categoría informativa en la que aparecen.

Observamos cómo los términos con mayor frecuencia de aparición se encuadran en el grupo de 
Tabla 4. Frecuencia de unidades informativas de las categorías C y D con términos pertenecientes a cada agrupación y porcentajes (entre paréntesis) con respecto a cada categoría y en total

\begin{tabular}{|c|c|c|c|c|}
\hline Grupo & Ejemplos & $\begin{array}{c}\text { Categorías C + D } \\
\mathrm{N}=254 \\
(\%)\end{array}$ & $\begin{array}{c}\text { Categoría C } \\
\mathrm{N}=135 \\
(\%)\end{array}$ & $\begin{array}{c}\text { Categoría D } \\
\mathrm{N}=119 \\
(\%)\end{array}$ \\
\hline Términos de la familia lingüística de la locura & Loco/a/s. enloquecer. locamente. & $\begin{array}{c}117 \\
(46.1)\end{array}$ & $\begin{array}{c}59 \\
(43.7)\end{array}$ & $\begin{array}{c}58 \\
(48.7)\end{array}$ \\
\hline $\begin{array}{l}\text { Términos relacionados con los síntomas positivos } \\
\text { de la esquizofrenia }\end{array}$ & $\begin{array}{l}\text { Delirar. alucinar. complejo de persecu- } \\
\text { ción }\end{array}$ & $\begin{array}{c}41 \\
(16.1)\end{array}$ & $\begin{array}{c}37 \\
(27.4)\end{array}$ & $\begin{array}{c}4 \\
(3.4)\end{array}$ \\
\hline Antónimos de locura/ loco & Lúcido. cuerdo. cabal. sano juicio & $\begin{array}{c}30 \\
(11.8)\end{array}$ & $\begin{array}{c}17 \\
(12.6)\end{array}$ & $\begin{array}{c}13 \\
(10.9)\end{array}$ \\
\hline $\begin{array}{l}\text { Términos populares para la enfermedad mental cróni- } \\
\text { ca. generalmente peyorativos }\end{array}$ & $\begin{array}{l}\text { Tarado. chiflado. chalado. poseso. } \\
\text { desequilibrado }\end{array}$ & $\begin{array}{c}22 \\
(8.7)\end{array}$ & $\begin{array}{c}8 \\
(5.9)\end{array}$ & $\begin{array}{c}14 \\
(11.8)\end{array}$ \\
\hline $\begin{array}{l}\text { Otros términos relacionados con problemas psicoló- } \\
\text { gicos }\end{array}$ & Demencia. obsesión. histeria. autolesión & $\begin{array}{c}21 \\
(8.3)\end{array}$ & $\begin{array}{c}10 \\
(7.4)\end{array}$ & $\begin{array}{c}11 \\
(9.2)\end{array}$ \\
\hline $\begin{array}{l}\text { Términos genéricos para designar enfermedad/ } \\
\text { enfermo mental }\end{array}$ & $\begin{array}{l}\text { Desequilibrio/ alteración/trastorno } \\
\text { Psíquico o mental }\end{array}$ & $\begin{array}{c}19 \\
(7.5)\end{array}$ & $\begin{array}{c}0 \\
(.0)\end{array}$ & $\begin{array}{c}19 \\
(16.0)\end{array}$ \\
\hline $\begin{array}{l}\text { Términos diagnósticos relacionados con enfermedad } \\
\text { mental crónica }\end{array}$ & Esquizofrenia. paranoia. psicosis & $\begin{array}{l}16 \\
(6.3)\end{array}$ & $\begin{array}{c}13 \\
(9.6)\end{array}$ & $\begin{array}{c}3 \\
(2.5)\end{array}$ \\
\hline $\begin{array}{l}\text { Términos relacionados con psicopatías (o trastornos } \\
\text { de personalidad) }\end{array}$ & $\begin{array}{l}\text { Psicópata. trastorno antisocial de la } \\
\text { personalidad }\end{array}$ & $\begin{array}{c}10 \\
(3.9)\end{array}$ & $\begin{array}{l}2 \\
(1.5) 0\end{array}$ & $\begin{array}{c}8 \\
(6.7)\end{array}$ \\
\hline Sinónimos de locura/ alocado & Insensatez. sinrazón. disparate & $\begin{array}{c}9 \\
(3.5)\end{array}$ & $\begin{array}{c}7 \\
(5.2)\end{array}$ & $\begin{array}{c}2 \\
(1.7)\end{array}$ \\
\hline Sinónimos de enloquecer & Perder la cabeza. enajenarse & $\begin{array}{c}8 \\
(3.1)\end{array}$ & $\begin{array}{c}2 \\
(1.5)\end{array}$ & $\begin{array}{c}6 \\
(5.0)\end{array}$ \\
\hline Términos relacionados con instituciones & Centro psiquiátrico. manicomio & $\begin{array}{c}5 \\
(2.0)\end{array}$ & $\begin{array}{c}1 \\
(0.7)\end{array}$ & $\begin{array}{c}4 \\
(3.4)\end{array}$ \\
\hline Términos relacionados con otros síntomas psicóticos & Estupor depresivo. despersonalización & $\begin{array}{c}5 \\
(2.0)\end{array}$ & $\begin{array}{c}4 \\
(3.0)\end{array}$ & $\begin{array}{c}1 \\
(0.8)\end{array}$ \\
\hline Términos relacionados con el tratamiento & Tratamiento/asistencia psiquiátrico & $\begin{array}{c}4 \\
(1.6)\end{array}$ & $\begin{array}{c}2 \\
(1.5)\end{array}$ & $\begin{array}{c}2 \\
(1.7)\end{array}$ \\
\hline $\begin{array}{l}\text { Términos relacionados con procedimientos psiquiátri- } \\
\text { cos }\end{array}$ & Electroshock. lobotomía & $\begin{array}{c}3 \\
(1.2)\end{array}$ & $\begin{array}{c}1 \\
(0.7)\end{array}$ & $\begin{array}{c}2 \\
(1.7)\end{array}$ \\
\hline Términos relativos a psicofármacos & Antipsicótico. neuroléptico. & $\begin{array}{c}3 \\
(1.2)\end{array}$ & $\begin{array}{c}2 \\
(1.5)\end{array}$ & $\begin{array}{c}1 \\
(0.8)\end{array}$ \\
\hline Otros & Salud/estado mental. informes psíquicos & $\begin{array}{c}10 \\
(3.9)\end{array}$ & $\begin{array}{c}1 \\
(0.7)\end{array}$ & $\begin{array}{c}9 \\
(7.6)\end{array}$ \\
\hline
\end{tabular}

Nota: C= Unidades informativas que incluyen términos relacionados con enfermedad mental crónica aplicados a situaciones u objetos; D= Incluyen términos relacionados con enfermedad mental crónica aplicados a personas o versan sobre enfermedad mental crónica.

"términos de la familia lingüística de la locura", seguido por el grupo de "términos relacionados con los síntomas positivos de la esquizofrenia”, que no suelen aparecer asociados a personas o en informaciones relativas a la enfermedad mental crónica (representan el $2.4 \%$ en la categoría D) sino aplicado a situaciones u objetos (27.4\% en la categoría $\mathrm{C}$ ). El tercer grupo de palabras más habituales lo ocupan "antónimos de la locura" y a continuación se sitúa el grupo de "términos populares para la enfermedad mental crónica, generalmente peyorativos", con una destacable presencia del $8.7 \%$.

Por tanto, el análisis de la terminología empleada por los medios de comunicación revela la utilización de expresiones mayoritariamente generales e inespecíficas, cuando no peyorativas. Existe una notable tendencia a incluir términos técnicos con significado negativo o como recurso literario, siendo escasa la aparición de titulares sobre la enfermedad mental con contenido positivo.

\section{Ubicación por secciones}

Respecto a la ubicación en la prensa escrita de las unidades informativas con contenidos relacionados con la enfermedad mental crónica (categorías $\mathrm{C}$ y D), se observa que el $38 \%$ de este tipo de información se encuadra en las secciones generales (Internacional, Madrid y el mundo), porcentaje similar al $32.7 \%$ incluido en las secciones de cultura y deportes. Se puede deducir un uso excesivo de 
este tipo de terminología, que contrasta con el $4.9 \%$ de unidades informativas localizadas en salud y sociedad, secciones teóricamente más vinculadas a la enfermedad mental. El resto de informaciones se encuadran en la sección de opinión $(15.1 \%)$ u otras ubicaciones $(9.3 \%)$.

\section{Temática de las unidades informativas}

Una revisión de las temáticas que tratan aquellas unidades informativas que contienen términos o expresiones relacionadas con enfermedad mental crónica aparece en la tabla 5.

Llama la atención constatar que, a pesar de la relativa frecuencia de uso de términos relacionados con enfermedad mental en los medios de comunicación, la proporción de unidades informativas que realmente se centran en estos contenidos es inferior a 1 de cada 1800. Tan sólo seis noticias del conjunto de unidades informativas analizadas conforman este grupo. Cuatro de éllas pertenecen a la prensa escrita y dos a programas televisivos, recibiendo un planteamiento ciertamente negativo: dos de las noticias relacionaban la enfermedad mental con el uso de drogas, una con delitos de violación, otra con pri- siones y dos eran denuncias por mala práctica y escasez de recursos para el tratamiento.

El examen de los temas planteados por las unidades informativas de las categorías $\mathrm{C}$ y $\mathrm{D}$, muestra que éstas mayoritariamente tienen relación con manifestaciones culturales y deportivas (36.6\%) y con materia política $(24.4 \%)$, resultando significativo el hecho de que el $15.3 \%$ de noticias se refieran a actos violentos y/o delictivos (fundamentalmente asesinato) aludiendo a la presencia o ausencia de problemas mentales en el agresor.

\section{Valoración de los jueces}

La presencia de estereotipos e información errónea y estigmatizante en las unidades informativas de las categorías C y D, se ha establecido en función de las valoraciones de los jueces según un criterio laxo que parte de un acuerdo mínimo de cinco jueces. Es decir, cuando al menos cinco jueces valoraron afirmativamente una variable para una determinada unidad informativa, se consideró que en dicha unidad informativa aparecía la variable.

Según el criterio descrito, la tabla 6 refleja el número de unidades informativas en las que los jue-

Tabla 5. Temática de las unidades informativas de las categorías C y D. Frecuencias y porcentajes (entre paréntesis) sobre el total de unidades informativas

\begin{tabular}{|c|c|c|c|}
\hline Temática de la unidad informativa & $\begin{array}{c}\mathbf{N}^{0} \text { de unidades } \\
\text { informativas } \mathrm{C}(\%)\end{array}$ & $\begin{array}{c}\mathrm{N}^{0} \text { de unidades } \\
\text { informativas D }(\%)\end{array}$ & $\begin{array}{c}\mathrm{N}^{0} \text { de unidades } \\
\text { informativas C y } \mathrm{D}(\%)\end{array}$ \\
\hline Enfermedad mental crónica & $0(\mathbf{0 . 0})$ & $6(5.0)$ & $6(2.4)$ \\
\hline Actos violentos y/o delictivos & $1(0.7)$ & $38(32.0)$ & $39(15.3)$ \\
\hline Manifestaciones culturales y deportivas & $57(42.2)$ & $36(30.3)$ & $93(36.6)$ \\
\hline Argumento de una obra & $15(11.1)$ & $10(8.4)$ & $25(9.8)$ \\
\hline Estreno o crítica de una obra & $16(11.9)$ & $4(3.4)$ & $20(7.9)$ \\
\hline Manifestación deportiva o taurina & $12(8.9)$ & $8(6.7)$ & $20(7.9)$ \\
\hline Declaraciones de un artista o deportista & $6(4.4)$ & $7(5.9)$ & $13(5.1)$ \\
\hline Cervantes y El Quijote & $8(5.9)$ & $7(5.9)$ & $15(5.9)$ \\
\hline Política & $41(30.4)$ & $21(17.6)$ & $62(24.4)$ \\
\hline Caso del Hospital Severo Ochoa & $14(10.4)$ & $5(4.2)$ & $19(7.5)$ \\
\hline Euskadi y las elecciones vascas & $10(7.4)$ & $3(2.5)$ & $13(5.1)$ \\
\hline Crisis política en Ecuador & $2(1.5)$ & $8(6.7)$ & $10(3.9)$ \\
\hline Otros temas políticos & $15(11.1)$ & $5(4.2)$ & $20(7.9)$ \\
\hline Otros temas de actualidad & $28(20.7)$ & $11(9.2)$ & $39(15.4)$ \\
\hline Elección papal & $10(7.4)$ & $2(1.7)$ & $12(4.7)$ \\
\hline Pisos de $3^{\circ} \mathrm{m} 2$ & $3(2.2)$ & $1(0.8)$ & $4(1.6)$ \\
\hline Otros temas & $15(11.1)$ & $8(6.7)$ & $23(9.1)$ \\
\hline Opinión & $8(5.9)$ & $7(5.9)$ & $15(5.9)$ \\
\hline TOTAL & $135(100)$ & $119(100)$ & $254(100)$ \\
\hline
\end{tabular}

Nota: C= Unidades informativas que incluyen términos relacionados con enfermedad mental crónica aplicados a situaciones u objetos; D= Incluyen términos relacionados con enfermedad mental crónica aplicados a personas o versan sobre enfermedad mental crónica. 
Tabla 6. Porcentaje de unidades informativas de las categorías C y D calificadas afirmativamente en cada variable por acuerdo entre los jueces

\begin{tabular}{lccc}
\hline Presencia de: & $\begin{array}{c}\text { \% unidades informativas } \\
\text { categorias C } \mathbf{~ D ~} \\
(\mathbf{n}=\mathbf{2 5 4})\end{array}$ & $\begin{array}{c}\text { \% unidades informativas } \\
\text { categoria C } \\
(\mathbf{n}=\mathbf{1 1 9})\end{array}$ & $\begin{array}{c}\text { \% unidades informativas } \\
\text { categoria } \mathbf{D} \\
(\mathbf{n = 1 3 5})\end{array}$ \\
\hline Estereotipo de peligrosidad & $12.6^{* * *}$ & 3 & 23.5 \\
Estereotipo de responsabilidad & .8 & 0 & 1.7 \\
Estereotipo de impredecibilidad & $9.1 * * *$ & 3 & 16 \\
Estereotipo incompetencia & 5.9 & 522 & 6.7 \\
Estigma & $25^{* * *}$ & 10.6 & 41.1 \\
Información errónea & 2.8 & 3 & 2.5 \\
\hline
\end{tabular}

$* * * \mathrm{p}<.001$ (Chi-cuadrado)

Nota: $\mathrm{C}=$ Unidades informativas que incluyen términos relacionados con enfermedad mental crónica aplicados a situaciones u objetos; D= Incluyen términos relacionados con enfermedad mental crónica aplicados a personas o versan sobre enfermedad mental crónica.

ces encontraron la presencia de información incorrecta, estereotipos o estigmatización, así como el porcentaje que representan dentro del total de 254 unidades informativas analizadas que contienen términos relativos a enfermedad mental crónica.

Los resultados del análisis Chi cuadrado son significativos para los dos estereotipos con mayor frecuencia de aparición, a saber, peligrosidad $\left(\chi^{2}(1)=24,3\right.$, $\mathrm{p}<.001)$ e impredecibilidad $\left(\chi^{2}(1)=12,99, \mathrm{p}<\right.$ $.001)$, así como para la presencia de estigma $\left(\chi^{2}(1)=\right.$ $31,18, \mathrm{p}<.001)$. En ambos casos, las cifras superiores en las unidades informativas de la categoría $\mathrm{D}$.

Es de señalar que la mayor parte de unidades informativas (79.5\%) no incluyen ninguno de estos estereotipos. En el resto se hayan presente uno (13\%) o dos estereotipos (7.1\%), existiendo tan sólo una unidad informativa en la que coincidían tres estereotipos y ninguna que incluyese los cuatro.

Un análisis detallado en función del medio de comunicación puede encontrarse en la tabla 7. Así, podemos observar la preponderancia de la televisión en la parición de los estereotipos de peligrosidad, responsabilidad e impredecibilidad, mientras que la radio ha sido el medio en que más frecuentemente se ha encontrado el estereotipo de incompetencia, información errónea sobre enfermedad mental y contenidos estigmatizantes. Los resultados de un análisis chi-cuadrado arrojaron diferencias estadísticamente significativas en función del medio para todas las variables consideradas.

En el caso concreto de la televisión, se consideró interesante realizar una comparación de los programas informativos con aquellos específicamente dedicados a sucesos (Gente y Sucedió en Madrid).

$\mathrm{Si}$ bien las conclusiones se ven limitadas por el escaso tamaño muestral del segundo grupo $(n=3)$, parece observarse una tendencia a la mayor presencia de estereotipos, información errónea y estigma en los programas de sucesos, donde los datos son superiores en todas las variables excepto el estereotipo de responsabilidad y el de incompetencia. No obstante, las diferencias encontradas en la prueba no paramétrica Kolmogorov-Smirnov no alcanzan la significación estadística.

\section{Discusión}

Los medios de comunicación son una de las principales fuentes de conocimiento sobre los trastornos

Tabla 7. Porcentajes de la presencia de estereotipos, estigma e información errónea en función del medio de comunicación

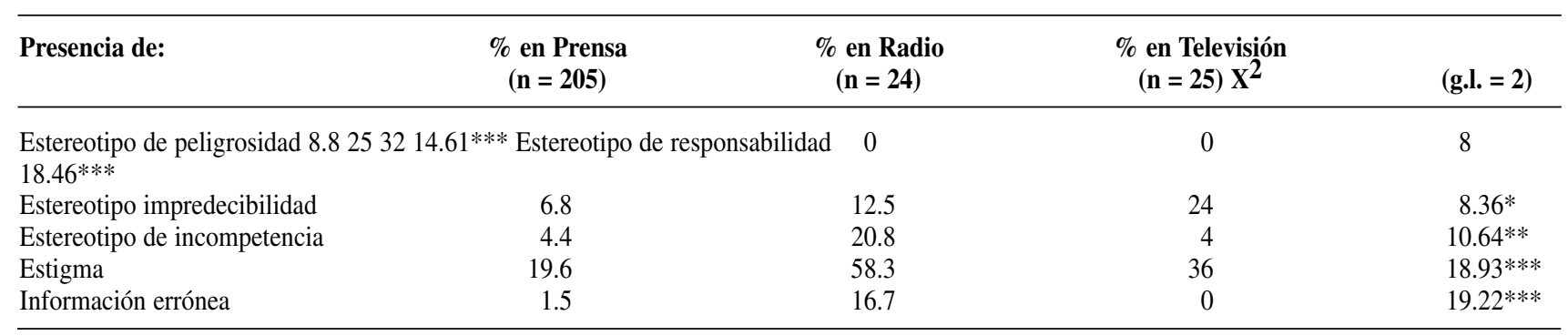

$* \mathrm{p}<.05 \quad * * \mathrm{p}<.01 \quad * * * \mathrm{p}<.001$ 
mentales a disposición de los ciudadanos (Cutcliffe y Hannigan, 2001; Organización Mundial de la Salud, 2001). Dada su capacidad de influencia, es esencial conocer hasta qué punto medios como la televisión, la prensa escrita o la radio pueden estar fomentando la transmisión de los estereotipos, actitudes y prejuicios negativos frecuentemente asociados a la enfermedad mental crónica.

Los resultados obtenidos por este estudio no se diferencian en gran medida de los hallados en otros contextos culturales, aunque presentan algunas matizaciones interesantes en nuestro entorno.

Estos resultados muestran un contraste entre la escasa presencia de información relacionada con enfermedad mental crónica en los principales medios de comunicación de nuestro país y la utilización abusiva de términos relacionados con patología mental. Tales términos o expresiones a menudo se utilizan de forma incorrecta o con un tono peyorativo y éticamente inaceptable para referirse a personas, objetos y situaciones, lo cual puede ayudar considerablemente a perpetuar el estigma social de los trastornos mentales.

Es interesante reparar en el hecho de que la mayoría de informaciones de los periódicos que incluyen expresiones relacionadas con trastornos mentales se ubican en secciones generales y de Cultura y Deportes, en lugar de en las secciones de Salud y Sociedad como cabría esperar. De hecho, la temática predominante se centra en manifestaciones culturales, deportivas y políticas, mientras que el tratamiento de información directamente relacionada con la salud mental es sumamente exiguo (uno de cada 1800 noticias en los periódicos abarca este tipo de contenidos) y a menudo se vincula con actos violentos o delictivos u otros contenidos de carácter negativo.

En la línea de otros estudios (Angermeyer y Schulze, 2001; Coverdale et al., 2002; Whal, Word y Richards, 2002), esta investigación halló una importante asociación, presente en los medios de comunicación, entre enfermedad mental crónica y violencia y peligrosidad. De hecho, los estereotipos de peligrosidad e impredecibilidad fueron los más frecuentemente encontrados (especialmente en las noticias referidas a personas) seguidos, en menor medida, por los de incompetencia e irresponsabilidad.

Si bien, tal y como encontraron Nairn y cols. (2001) en Nueva Zelanda, nuestro trabajo permite concluir que los medios raramente ofrecen información errónea sobre los trastornos mentales, la aparición de estereotipos y el tratamiento generalmente negativo de la información propician la transmisión del estigma social. En concreto, un $25 \%$ de las informaciones analizadas en este trabajo resultaron estigmatizadoras.

A pesar de que las diferencias entre medios de comunicación son escasas, los resultados apuntan a un mejor tratamiento del tema de la enfermedad mental en la prensa escrita. La televisión destaca por trasmitir con mayor frecuencia los estereotipos de peligrosidad, responsabilidad e impredecibilidad, especialmente en los programas dedicados de forma específica a los sucesos. Por su parte, las informaciones emitidas en la radio han resultado contener en mayor medida el estereotipo de incompetencia, información errónea y contenidos estigmatizantes.

No obstante, la comparación entre medios específicos (periódicos, emisoras de radio o cadenas televisivas concretas) no arrojó diferencias significativas, por lo que se debe entender que existe una similaridad en el tratamiento informativo del tema.

La interpretación de los resultados exige tomar en consideración el hecho de que este trabajo se centra en espacios de carácter informativo, excluyendo contenidos de ficción y entretenimiento de la radio y televisión, cuya influencia en la difusión del estigma social también sería interesante examinar. Respecto a la prensa escrita, se propone incluir en futuros estudios los periódicos de distribución gratuita, debido a sus altas tasas de consumo y las características diferenciales respecto a los diarios aquí analizados.

Del mismo modo en que los medios de comunicación influyen en la perpetuación del estigma, con las limitaciones y sufrimiento que ello supone para las personas afectadas por un trastorno mental crónico, el tratamiento adecuado y riguroso del tema por parte de estos mismos medios podría tener un papel fundamental en la lucha contra los prejuicios y la discriminación. 


\section{Extended Summary}

\section{Introduction}

Social perception of mental illness is characterized by a wide range of negative stereotypes and prejudices which lead to discrimination and increase the suffering in people with a mental disorder. (Muñoz Perez-Santos, Crespo \& Guillén, 2009). The stereotypes usually include information about: their dangerousness and relation with violent acts; their responsibility either for the illness itself or for not having been capable of curing it by treatment; their weak character; their incompetence and incapacity to carry out essential tasks such as self-care; their unpredictable character and reactions, and their lack of control. (Crespo, Muñoz, Pérez-Santos and Guillén, 2008). When prejudices are activated, these beliefs can lead to reactions of fear, concern, distrust... which, in turn, can trigger various forms of discrimination that place people with a mental disorder in social disadvantage. In short, stereotypes, prejudice and discrimination associated with mental illness may deprive of opportunities essential to achieving their life goals, especially those that deal with economic and personal independence (Corrigan, 2005; Thornicroft, 2006).

The role of mass media is essential to understand the maintenance of the stigmatization of people with mental disorders in our society (Cuenca, 2003). In fact, some authors emphasize the responsibility of the media in perpetuating stereotypes, prejudices and discriminatory practices, due to the negative and inaccurate representation that they make of mental disorders (Cutcliffe and Hannigan, 2001).

The representation of mental illness in the press is predominantly negative (eg, Coverdale et al. 2002; Nairn, Coverdale, and Claasen, 2001; Walha et al., 2002), appearing frequently linked to violent and criminal events (eg, Angermeyer and Schulze, 2001; Coverdale et al, 2002; Gamo, 1992, Huang and Test, 2003; Instituto Andaluz de Salud Mental, 1987). The negative aspects are usually highlighted, with a scarcity of information about people with mental disorder who have a normal life. This vision is reflecting the stereotypes of dangerous- ness, unpredictability, lack of control or vulnerability.

This paper attempts to examine the treatment given to mental illness in the informative spaces in the media in our country and the possible presence of stigmatizing tendencies. Thus, it is intended to compensate in some degree the lack of such studies in our sociocultural context as well as the limited number of studies that have examined various mass media at the same time (Francis et al., 2004, Francis et al., 2005).

\section{Method}

\section{Procedure of sampling}

\section{Media Select}

This study aims to analyze the information coming mostly to citizens, so the selection of the media was made based on data from the General Media for the period between April 2004 and March 2005 (Asociación para la investigación de Medios de Comunicación, 2005). The analysis included newspapers, radio and television, as they were the most used mass media by the public.

\section{Sampling period}

The study was conducted on the information transmitted for 14 consecutive days, from Monday $11^{\text {th }}$ to Sunday April 24th, 2005.

\section{Unit of analysis}

The unit of analysis used was the "unit of information" or any oral or written text preceded by heading (that includes various types information such as news, interviews, opinion pieces, etc). The primary outcome measure was the frequency of unit of informations, as well as duration in the case of radio and television. 


\section{Contents included in the sampling}

All in all 7.532 written unit of informations (from newspapers), 3.079,34 radio minutes and 2.284,89 TV emission minutes were analyzed.

\section{Data analysis}

\section{Classification of unit of informations}

Each unit of information was classified into five categories: Category 0 (unrelated to mental health; Originally selected but ruled out in the second stage of analysis); Category A (Unit of informations including psychological terms applied to situations or objects); Category B (Unit of informations including psychological terms applied to individuals or groups, as well as general information on mental health); Category $\mathrm{C}$ (Unit of informations including words or phrases associated with chronic mental illness applied to situations or objects); Category D (Unit of informations including terms and expressions relating to chronic mental illness applied to individuals or groups and unit of informations on chronic mental illness).

Frequency analysis: We performed a descriptive analysis of global frequencies (number of unit of informations assigned to each category from every newspaper, radio and television) and an analysis of frequencies depending on each medium.

Content analysis: We examined the specific content of the unit of informations that included words or phrases associated with chronic mental illness (categories C and D) and, therefore, represent the main focus of this study. In this regard, we applied the following scheme of analysis: a) Analysis of the terms used; b) Analysis of the sections where these unit of informations appear; c) Thematic analysis of the unit of informations; d) Analysis of each unit of information by independent judges.

\section{Results}

\section{Results of frequency analysis}

\section{Global frequency}

The total sum of unit of informations in the papers is 625 units, 409 if we exclude those in cate- gory 0 , which is ruled out for including psychological terms without reference to mental health. There are 50 unit of informations for the case of radio and 57 for television. The majority of units are included within the category B.

\section{Frequencies in the press}

Among the 7.532 unit of informations analyzed in the newspapers, 409 belong to categories A, B, C or $\mathrm{D}$, which means that $5.43 \%$ of stories examined include a psychological term or expression of ordinary language commonly used to refer to mental health of people. The percentage of unit of informations referred to severe and chronic mental illness stands at $2.72 \%$.

\section{Radio frequencies}

Among the 50 news that included references to mental health (only $2.2 \%$ of total broadcasting time), there were 24 that specifically contained terms related to severe and chronic mental illness. Most of the time was occupied by units from category D.

\section{Television frequencies}

The amount of time occupied by contents in categories A, B, C and D represents $4.24 \%$ of total analyzed time. The percentage drops to $2.05 \%$ when considering only those unit of informations that make specific reference to severe and chronic mental disorders. The percentages are higher with regard to programs of events: they spend $22 \%$ of time to broadcast units including a psychological term or expression of ordinary language commonly used to refer to mental health. In $11.55 \%$ of total time, issues related to chronic mental illness are addressed.

\section{Results of content analysis}

Terminology

The term most frequently occurring is "crazy" as applied to objects, situations or people (107 cases), followed by "madness" (48 appearances) and "delirium" (28 times). 


\section{Location of sections}

Results show that $38 \%$ of the unit of informations related to chronic mental illness (categories $\mathrm{C}$ and $\mathrm{D}$ ) appear in the general sections and $32.7 \%$ in the culture and sports sections. Only $4.9 \%$ of unit of informations are located in health and society, sections theoretically most closely linked to mental illness.

\section{Thematic of unit of informations}

Thematic mainly relates to cultural and sporting events $(36.6 \%)$ and politics $(24.4 \%)$. It is significant that $15.3 \%$ of news are related to violence and /or crime (basically murder), alluding to the presence or absence of mental illness in an aggressor.

Only six news of all the units analyzed include information really focus on mental illness, but certainly getting a negative approach (two of the news related mental illness with drug use, one with rape and imprisonment and two other complaints about malpractice and lack of resources for treatment).

\section{Rating judges}

According to judges' rating, the most common stereotype in categories $\mathrm{C}$ and $\mathrm{D}$ were dangerousness (12.6\% of the unit of informations) and unpredictability $(9.1 \%)$. Stigma was found in $25 \%$ of the unit of informations.

Nervertheless, most unit of informations (79.5\%) did not include any stereotypes There is a preponderance of television in the development of stereotypes of dangerousness, responsibility and unpredictability, while the stereotype of incompetence, misinformation about mental illness and stigmatizing content are more frequent in radio.

\section{Discussion}

Results show a contrast between the scarcity of information about chronic mental illness in the mainstream media in our country and the misuse of terms related to mental pathology. Such terms or expressions are often used incorrectly or with a pejorative tone to refer to people, objects and situations, which can significantly help to perpetuate the stigma of mental disorders.

Most newspaper reports which include statements relating to mental disorders are located in general sections or in culture and sports, rather than in health and society section as expected. In fact, the predominant theme focuses on cultural, sporting and politics, while the treatment of information directly related to mental health is very limited and is often linked with violent or criminal acts or with other negative contents.

In line with other studies (Angermeyer and Schulze, 2001; Coverdale et al. 2002; Wahl, Word and Richards, 2002), this study found a significant association between chronic mental illness and violence in the media. In fact, the stereotypes of dangerousness and unpredictability were the most frequently encountered, followed by the incompetence and irresponsibility.

Our work leads to the conclusion that, even though the media rarely offer misinformation about mental disorders, as Nairn et al. (2001) found in New Zealand, the emergence of stereotypes and negative treatment information generally perpetuate the transmission of social stigma. Specifically, $25 \%$ of the information analyzed in this work was stigmatizing.

Despite the differences between media are scarce, the results point to a better treatment of mental illness issues in print media. Television often broadcast stereotypes of dangerousness, unpredictability and responsibility, especially in programs devoted specifically to accidents and crime reports. Meanwhile, the information broadcasted on the radio highlighted the stereotype of incompetence, misinformation and stigmatizing content.

Just as the media influence in perpetuating the stigma, a proper and rigorous approach to mental illness news and references could have a crucial role in the fight against prejudices and discrimination.

\section{Referencias}

Angermeyer, M. C., Dietrich, S., Pott, D. y Matschinger, H. (2005). Media consumption and 
desire for social distance towards people with schizophrenia. European Psychiatry, 20, 246250.

Angermeyer, M. C. y Schulze, B. (2001). Reinforcing stereotypes: How to focus on forensic cases in news reporting may influence public attitudes towards the mentally ill. International Journal of Law and Psychiatry, 24, 569-486.

Arceo, J. L. (2005, Julio). Presentación del informe "Tratamiento de la Esquizofrenia en la prensa española, 2003-2004", Conferencia en los Cursos de Verano de El Escorial, 14-07-05.

Asociación Española de Neuropsiquiatría. (2007). Consenso sobre promoción de la salud mental, prevención del trastorno mental y disminución del estigma. Madrid: Asociación española de neuropsiquiatría.

Asociación para la investigación de los medios de Comunicación. (2005). Encuesta General de Medios. Primer año móvil. Madrid: Asociación para la investigación de Medios de Comunicación.

Corrigan, P. W. (2005). On the stigma of mental illness: Practical strategies for research and social change. Washington DC: American Psychological Association Press.

Coverdale, J., Nairn, R. y Claasen, D. (2002). Depictions of mental illness in print media: A prospective national sample. Australian and New Zealand Journal of Psychiatry, 36, 697-700.

Crespo, M., Muñoz, M., Pérez Santos, E. y Guillén, A. I. (2008) Stigma associated with severe and persistent mental illness among the general population of Madrid (Spain): descriptive study. Community Mental Health Journal, 44, 393-403

Crocker, J., Major, B. y Steele, C. (1998). Social Stigma. In D. T. Gilbert (Ed.), The Handbook of Social Psychology. Boston, MA: McGraw-Hill.

Cuenca, O. (2003). El estigma de la enfermedad mental en los medios de comunicación. En J. J. López-Ibor, J. C. Gómez y J. A. Gutiérrez (Eds.), Retos para la Psiquiatría y la Salud Mental en España. Madrid: Ars Medica.

Cutcliffe, J. R. y Hannigan, B. (2001) Mass Media, "monsters" and mental health clients: The need for increased lobbying. Journal of Psychiatric and Mental Health Nursing, 8, 315-321.
Depla, M., de Graaf, R., van Weeghel, J. y Heeren, T. J. (2005). The role of stigma in the quality of life of older adults with severe mental illness. International Journal of Geriatric Psychiatry, 20, 146-153.

Dickerson, F. B., Sommerville, J., Origoni, A. E., Ringel, N. B. y Parente, F. (2002). Experiences of stigma among outpatients with schizophrenia. Schizophrenia Bulletin, 28, 143-156.

Ertugrul, A. y Ulug, B. (2004). Perception of stigma among patients with schizophrenia. Social Psychiatry and Psychiatric Epidemiology, 39, 7377.

Francis, C., Pirkis, J., Blood, R. W., Dunt, D., Burgess, P., Morley, B., ... Putnis, P. (2004). The portrayal of mental health and illness in Australian nonfiction media. The Australian and New Zealand Journal of Psychiatry, 38, 541-546.

Francis, C., Pirkis, J., Blood, R. W., Dunt, D., Burgess, P., Morley, B., et al. (2005). Media portrayal of depression and other mental illnesses in Australian. Journal of Community Psychology, 33, 283-297.

Gamo, E. (1992). Información sobre salud mental en la prensa. Su repercusión en la educación sanitaria. Revista de la Asociación Española de Neuropsiquiatría, 12, 43-57.

Huang, B. y Priebe, S. (2003). Media coverage of mental health care in UK, USA and Australia. Psychiatric Bulletin, 27, 331-333.

Instituto Andaluz de Salud Mental de Andalucía. (1987). Salud mental y prensa. Consejería de Salud. Junta de Andalucía.

Lawson, A. y Fouts, G. (2004). Mentall Illness in Disney animated films. Canadian Journal of Psychiatry, 49, 310-314.

Link, B. G. y Phelan, J. C. (2001). Conceptualizing stigma. Annual Review of Sociology, 27, 363-385.

Link, B. G., Struening, E. L., Neese, T. S., Asmussen, S. y Phelan, J. C. (2001). Stigma as a barrier to recovery: The consequences of stigma for the self-steem of people with mental illnesses. Psychiatric Services, 52, 1621-1626.

Link, B. G., Struening, E. L., Rahav, M., Phelan, J. C., et al. (1997). On stigma and its consequences: Evidence from a longitudinal study of men with dual diagnoses of mental illness and substance 
abuse. Journal of Health and Social Behavior, 38, 177-190.

López, M., Laviana M., Fernández, L., López, A., Rodríguez, A. M. y Aparicio, A. (2008). La lucha contra el estigma y la discriminación en salud mental: Una estrategia compleja basada en la información disponible. Revista de la Asociación Española de Neuropsiquiatría, 101, 43-83.

Markowitz, F. E. (1998). The effects of stigma on the psychological well-being and life satisfaction of persons with mental illness. Journal of Health and Social Behavior, 39, 335-347.

Minnebo, J. y Van Acker, A. (2004). Does television influence adolescents' perceptions of and attitudes toward people with mental illness? Journal of Community Psychology, 32, 257-275.

Muñoz, M., Pérez-Santos, E., Crespo, M. y Guillén, A.I. (2009). El estigma de la enfermedad mental. Madrid: Ed. Complutense.

Nairn, R., Coverdale, J. y Claasen, D. (2001). From source material to news story in New Zealand print media: A prospective study of the stigmatizing processes in depicting mental illness. Australian and New Zealand Journal of Psychiatry, 35, 654-659.

Organización Mundial de la Salud. (2001). Informe sobre la Salud en el mundo 2001. Salud mental: Nuevos conocimientos, nuevas esperanzas. Ginebra: Organización Mundial de la Salud.

Ottati, V., Bodenhausen, G. V. y Newman, L. S. (2004). Social psychological models of mental illness stigma. En P. W. Corrigan (Ed), On Stigma of Mental Illness (pp. 99-128). Washington D. C.: American Psychological Association.
Ritsher, J. B. y Phelan, J. C (2004). Internalized stigma predicts erosion of morale among psychiatric outpatients. Psychiatry Research, 129, 257265.

Rosenfield, S. (1997) Labelling mental Illness: The effects of received services and perceived stigma on life satisfaction. American Sociological Review, 62, 660-672.

Sieff, E. M. (2003). Media frames of mental illnesses: The potential impact of negative frames. Journal of Mental Health (UK). 12, 259-269.

Signorelli, N. (1989). The stigma of mental illness on television. Journal of Broadcasting and Electronic Media, 33, 325-331.

Thornicroft, G. (2006) Shunned: Discrimination against People with Mental Illness. Oxford University Press.

Vaughan, G. y Hensen, C. (2004). "Like minds, like mine": A New Zeland project to counter the stigma and discrimination associated with mental illness. Australasian psychiatry: bulletin of Royal Australian and New Zealand College of Psychiatrists, 12, 113-117.

Wahl, O. (1999). Mental health consumers' experience of stigma. Schizophrenia Bulletin, 25, 467478.

Wahl, O., Wood, A. y Richards, R. (2002). Newspaper coverage of mental illness: Is it changing? Psychiatric Rehabilitation Skills, 6, 931.

Wahl, O., Wood, A., Zaveri, P., Drapalski, A. y Mann, B. (2003). Mental illness depiction in children's films. Journal of Community Psychology, 31, 553-556.

Manuscrito recibido:09/02/2011

Revisión recibida: 21/03/2011

Aceptado: 05/05/2011 